\title{
Positive solutions of nonlinear elliptic systems
}

\author{
by Robert Dalmasso (Grenoble)
}

\begin{abstract}
We study the existence and nonexistence of positive solutions of nonlinear elliptic systems in an annulus with Dirichlet boundary conditions. In particular, $L^{\infty}$ a priori bounds are obtained. We also study a general multiple linear eigenvalue problem on a bounded domain.
\end{abstract}

1. Introduction. In this paper we investigate the existence and nonexistence of positive solutions of the nonlinear elliptic system

$$
\begin{cases}-\Delta u_{j}=f_{j+1}\left(u_{j+1}\right), \quad j=1, \ldots, m-1, & \text { in } \Omega(a, b), \\ -\Delta u_{m}=f_{1}\left(u_{1}\right) & \text { in } \Omega(a, b), \\ u_{j}=0, \quad j=1, \ldots, m, & \text { on } \partial \Omega(a, b),\end{cases}
$$

where $0<a<b<\infty, \Omega(a, b)$ denotes the annulus $\left\{x \in \mathbb{R}^{n}: a<|x|<b\right\}$ $(n \geq 2)$ and $m \geq 2$ is an integer. $u=\left(u_{1}, \ldots, u_{m}\right) \in\left(C^{2}(\overline{\Omega(a, b)})\right)^{m}$ is a positive solution of (1.1) if the functions $u_{j}$ satisfy (1.1) and $u_{j}>0$ on $\Omega(a, b)$ for $j=1, \ldots, m$.

When $m=2$, Ph. Clément, D. G. de Figueiredo and E. Mitidieri [2] considered the existence of positive solutions in convex domains and L. A. Peletier and R. C. A. M. van der Vorst [6] studied the case of a ball. In both cases the method used to prove the existence of a positive solution consisted of first obtaining a priori estimates on the positive solutions and then applying well-known properties of compact mappings taking a cone in a Banach space into itself (see D. G. de Figueiredo, P.-L. Lions and R. D. Nussbaum [3]).

Our first result is the following $L^{\infty}$ bound for positive radial solutions of problem (1.1).

THEOREM 1.1. Let $f_{j}, j=1, \ldots, m$, satisfy the following hypotheses:

$\left(\mathrm{H}_{1}\right) \quad f_{j}:[0, \infty) \rightarrow \mathbb{R}$ is a continuous function,

1991 Mathematics Subject Classification: 35B45, 35J55.

Key words and phrases: a priori bounds, nonlinear elliptic systems, Maximum Principle. 
$\left(\mathrm{H}_{2}\right) \quad \liminf \operatorname{in}_{u \rightarrow \infty} f_{j}(u) / u>\alpha_{j}>0$ and $\alpha_{1} \ldots \alpha_{m}>\mu_{1}^{m}$ where $\mu_{1}$ denotes the principal eigenvalue of $-\Delta$ on $\Omega(a, b)$ with Dirichlet boundary conditions.

Then there exists $M>0$ such that

$$
\left\|u_{j}\right\|_{\infty} \leq M, \quad j=1, \ldots, m
$$

for all positive radial solutions $\left(u_{1}, \ldots, u_{m}\right) \in\left(C^{2}(\overline{\Omega(a, b)})\right)^{m}$ of $(1.1)$.

Under some additional assumptions on the functions $f_{j}$, we can use Theorem 1.1 to establish the existence of a positive radial solution of problem (1.1).

Theorem 1.2. Let $f_{j}, j=1, \ldots, m$, satisfy $\left(\mathrm{H}_{1}\right),\left(\mathrm{H}_{2}\right)$. Assume moreover that

$\left(\mathrm{H}_{3}\right) \quad f_{j}(u) \geq 0$ for $u>0, j=1, \ldots, m$,

$\left(\mathrm{H}_{4}\right) \quad \lim \sup _{u \rightarrow 0} f_{j}(u) / u<\beta_{j}$ for $j=1, \ldots, m$ and $\beta_{1} \ldots \beta_{m}<\mu_{1}^{m}$.

Then problem (1.1) has at least one positive radial solution $\left(u_{1}, \ldots, u_{m}\right) \in$ $\left(C^{2}(\overline{\Omega(a, b)})\right)^{m}$.

We have the following nonexistence result.

THEOREM 1.3. Assume that one of the following conditions is satisfied:

(i) $f_{j}(s)>\gamma_{j} s$ for $s>0, j=1, \ldots, m$ where $\gamma_{1} \ldots \gamma_{m} \geq \mu_{1}^{m}$;

(ii) $f_{j}(s)<\delta_{j} s$ for $s>0, j=1, \ldots, m$ where $\delta_{j} \geq 0$ for $j=1, \ldots, m$ and $\delta_{1} \ldots \delta_{m} \leq \mu_{1}^{m}$.

Then problem (1.1) has no positive solution (whether radial or not) $\left(u_{1}, \ldots, u_{m}\right) \in\left(C^{2}(\overline{\Omega(a, b)})\right)^{m}$.

As an application we consider the problem

$$
\begin{cases}-\Delta u_{j}=\varrho_{j+1} u_{j+1}+u_{j+1}^{p_{j+1}}, \quad j=1, \ldots, m-1, & \text { in } \Omega(a, b), \\ -\Delta u_{m}=\varrho_{1} u_{1}+u_{1}^{p_{1}} & \text { in } \Omega(a, b), \\ u_{j}=0, \quad j=1, \ldots, m, & \text { on } \partial \Omega(a, b),\end{cases}
$$

where $\varrho_{j} \geq 0$ and $p_{j}>1$ for $j=1, \ldots, m$. For problem (1.2) we have the following corollary.

Corollary 1.1. (i) Assume that $\varrho_{1} \ldots \varrho_{m}<\mu_{1}^{m}$. Then problem (1.2) has at least one positive radial solution $\left(u_{1}, \ldots, u_{m}\right) \in\left(C^{2}(\overline{\Omega(a, b)})\right)^{m}$.

(ii) Assume that $\varrho_{1} \ldots \varrho_{m} \geq \mu_{1}^{m}$. Then problem (1.2) has no positive solution (whether radial or not) $\left(u_{1}, \ldots, u_{m}\right) \in\left(C^{2}(\overline{\Omega(a, b)})\right)^{m}$.

Rem ark 1.1. Problem (1.2) is an example of a perturbed system (see Theorem 2.1 in Section 2). 
When we are interested in positive radial solutions, the problem under consideration reduces to the one-dimensional boundary value problem

$$
\begin{cases}-\Delta u_{j}=f_{j+1}\left(u_{j+1}\right), \quad j=1, \ldots, m-1, & \text { in }(a, b), \\ -\Delta u_{m}=f_{1}\left(u_{1}\right) & \text { in }(a, b), \\ u_{j}(a)=u_{j}(b)=0, \quad j=1, \ldots, m, & \end{cases}
$$

where $\Delta$ denotes the polar form of the Laplacian, i.e.

$$
\Delta=t^{1-n} \frac{d}{d t}\left(t^{n-1} \frac{d}{d t}\right)
$$

Our paper is organized as follows. In Section 2 we study a general multiple linear eigenvalue problem for systems. Theorems 1.1, 1.2 and 1.3 are proved in Sections 3, 4 and 5 respectively. Finally, in Section 6 we give a qualitative result for positive solutions (whether radial or not) of (1.1) when the functions $f_{j}$ are nondecreasing.

2. A multiple linear eigenvalue problem for systems. In this section we consider the linear eigenvalue problem

$$
\begin{cases}-\Delta u_{j}=\lambda_{j+1} u_{j+1}, \quad j=1, \ldots, m-1, & \text { in } \Omega, \\ -\Delta u_{m}=\lambda_{1} u_{1} & \text { in } \Omega, \\ u_{j}>0, \quad j=1, \ldots, m, & \text { in } \Omega, \\ u_{j}=0, \quad j=1, \ldots, m, & \text { on } \partial \Omega,\end{cases}
$$

where $\Omega$ is a bounded domain in $\mathbb{R}^{n}(n \geq 1)$ with smooth boundary $\partial \Omega$ and $m \geq 2$ is an integer. We denote by $\mu_{1}$ the principal eigenvalue of the Laplacian on $\Omega$ with Dirichlet boundary conditions and $\varphi_{1}$ is the corresponding (positive) eigenfunction. We have the following theorem.

Theorem 2.1. Problem (2.1) has a solution if and only if

$$
\lambda_{j}>0, \quad j=1, \ldots, m, \quad \text { and } \quad \lambda_{1} \ldots \lambda_{m}=\mu_{1}^{m} .
$$

The solution is given by $u_{j}=c_{j} \varphi_{1}$ where $c_{1}>0$ is an arbitrary constant and $c_{j}=c_{1}\left(\lambda_{2} \ldots \lambda_{j}\right)^{-1}\left(\lambda_{1} \ldots \lambda_{m}\right)^{(j-1) / m}$ for $j=2, \ldots, m$.

Proof. We first note that $\lambda_{j}>0$ for $j=1, \ldots, m$. Indeed assume that there exists $j \in\{1, \ldots, m\}$ such that $\lambda_{j} \leq 0$. We deduce from (2.1) that $\Delta u_{j-1} \geq 0$ on $\Omega$ (where $u_{j-1}=u_{m}$ if $j=1$ ), hence $u_{j-1} \leq 0$ on $\Omega$ by the Maximum Principle and we reach a contradiction.

LEMMA 2.1. Let $a_{1}>0$ be arbitrary and define

$$
a_{j}=a_{1} \lambda_{2} \ldots \lambda_{j}\left(\lambda_{1} \ldots \lambda_{m}\right)^{(1-j) / m} \quad \text { for } j=2, \ldots, m .
$$

Then, setting $v_{j}=a_{j} u_{j}$, we have

$$
\begin{cases}-\Delta v_{j}=\mu_{1} v_{j+1}, \quad j=1, \ldots, m-1, & \text { in } \Omega, \\ -\Delta v_{m}=\mu_{1} v_{1} & \text { in } \Omega, \\ v_{j}=0, \quad j=1, \ldots, m, & \text { on } \partial \Omega .\end{cases}
$$


Moreover, $\mu_{1}^{m}=\lambda_{1} \ldots \lambda_{m}$.

P r o of. Using (2.1) we easily get

$$
\begin{cases}-\Delta v_{j}=\alpha v_{j+1}, \quad j=1, \ldots, m-1, & \text { in } \Omega, \\ -\Delta v_{m}=\alpha v_{1} & \text { in } \Omega, \\ v_{j}=0, \quad j=1, \ldots, m, & \text { on } \partial \Omega,\end{cases}
$$

where $\alpha=\left(\lambda_{1} \ldots \lambda_{m}\right)^{1 / m}$, from which we deduce that

$$
\begin{cases}-\Delta\left(v_{1}+\ldots+v_{m}\right)=\alpha\left(v_{1}+\ldots+v_{m}\right) & \text { in } \Omega, \\ v_{1}+\ldots+v_{m}=0, & \text { on } \partial \Omega .\end{cases}
$$

We have $v_{1}+\ldots+v_{m}>0$ on $\Omega$. Since the only positive solution of problem (2.3) is $C \varphi_{1}$ with the eigenvalue $\mu_{1}$ where $C>0$ is a constant, we get $v_{1}+\ldots+v_{m}=C \varphi_{1}$ with $C>0$ and $\alpha=\mu_{1}$. The proof of the lemma is complete.

To conclude the proof of the theorem we shall prove that

$$
v_{1}=\ldots=v_{m} .
$$

We prove (2.4) by induction.

Assume that $m=2$. Then from Lemma 2.1 we get

$$
\begin{cases}\Delta\left(v_{1}-v_{2}\right)=\mu_{1}\left(v_{1}-v_{2}\right) & \text { in } \Omega \\ v_{1}-v_{2}=0 & \text { on } \partial \Omega .\end{cases}
$$

Multiplying the differential equation of (2.5) by $v_{1}-v_{2}$ and integrating over $\Omega$ we obtain

$$
-\int_{\Omega}\left|\nabla\left(v_{1}-v_{2}\right)\right|^{2} d x=\mu_{1} \int_{\Omega}\left(v_{1}-v_{2}\right)^{2} d x
$$

which implies that $v_{1}=v_{2}$.

Now we suppose that (2.4) holds for $2 \leq m \leq r-1(r \geq 3)$ and we must prove it for $m=r$.

If $r=2 p \geq 4$, then from Lemma 2.1 we get

$$
(-1)^{p} \Delta^{p} v_{1}=\mu_{1}^{p} v_{p+1} \quad \text { and } \quad(-1)^{p} \Delta^{p} v_{p+1}=\mu_{1}^{p} v_{1} \quad \text { on } \Omega,
$$

from which we deduce

$$
(-1)^{p} \Delta^{p}\left(v_{1}-v_{p+1}\right)=-\mu_{1}^{p}\left(v_{1}-v_{p+1}\right) .
$$

Multiplying (2.6) by $v_{1}-v_{p+1}$ and integrating over $\Omega$ yields

$$
(-1)^{p} \int_{\Omega}\left(v_{1}-v_{p+1}\right) \Delta^{p}\left(v_{1}-v_{p+1}\right) d x=-\mu_{1}^{p} \int_{\Omega}\left(v_{1}-v_{p+1}\right)^{2} d x .
$$

The left hand side in (2.7) is equal to

$$
\int_{\Omega}\left(\Delta^{k}\left(v_{1}-v_{2 k+1}\right)\right)^{2} d x \quad \text { if } p=2 k,
$$


and to

$$
\int_{\Omega}\left|\nabla \Delta^{k}\left(v_{1}-v_{2 k+2}\right)\right|^{2} d x \quad \text { if } p=2 k+1 .
$$

In each case we get $v_{1}=v_{p+1}$. Then using the induction hypothesis we obtain $v_{1}=\ldots=v_{2 p}$.

If $r=2 p+1$, we write

$$
\begin{array}{r}
\sum_{1 \leq j<k \leq 2 p+1} \int_{\Omega}\left(v_{j}-v_{k}\right)^{2} d x=\sum_{1 \leq j<k \leq 2 p+1} \int_{\Omega}\left(v_{j}^{2}+v_{k}^{2}-2 v_{j} v_{k}\right) d x \\
=2 p \sum_{j=1}^{2 p+1} \int_{\Omega} v_{j}^{2} d x-2 \sum_{1 \leq j<k \leq 2 p+1} \int_{\Omega} v_{j} v_{k} d x .
\end{array}
$$

Now for each $i \in\{1, \ldots, 2 p+1\}$ we define

$$
\begin{aligned}
& A_{i}=\left\{(j, k): 1 \leq j<k \leq 2 p+1 \text { and } \int_{\Omega} v_{i}^{2} d x=\int_{\Omega} v_{j} v_{k} d x\right\}, \\
& B_{i}=\left\{h \in\{1, \ldots, 2 p+1\}: \int_{\Omega} v_{i}^{2} d x=\int_{\Omega} v_{h}^{2} d x\right\} .
\end{aligned}
$$

Let $s \in\{1, \ldots, 2 p+1\}$ be such that

$$
\{1, \ldots, 2 p+1\}=\bigcup_{a=1}^{s} B_{i_{a}} \quad \text { and } \quad B_{i_{a}} \cap B_{i_{b}}=\emptyset \quad \text { for } a \neq b .
$$

We shall show that

$$
\# A_{i_{a}}=\# B_{i_{a}} \cdot p \quad \text { for } a=1, \ldots, s .
$$

Then, since $\sum_{1 \leq j<k \leq 2 p+1} 1=p(2 p+1)$ we deduce from (2.8) and (2.9) that

$$
\sum_{1 \leq j<k \leq 2 p+1} \int_{\Omega}\left(v_{j}-v_{k}\right)^{2} d x=0
$$

and hence $v_{1}=\ldots=v_{2 p+1}$.

It remains to prove (2.9). Using the equations (2.2) and integrations over $\Omega$ we obtain

$$
\begin{aligned}
\int_{\Omega} v_{p+1}^{2} d x & =\int_{\Omega} v_{p} v_{p+2} d x=\ldots=\int_{\Omega} v_{1} v_{2 p+1} d x \\
\int_{\Omega} v_{j}^{2} d x & =\int_{\Omega} v_{j-1} v_{j+1} d x=\ldots=\int_{\Omega} v_{1} v_{2 j-1} d x \\
& =\int_{\Omega} v_{2 p+1} v_{2 j} d x=\ldots=\int_{\Omega} v_{p+j} v_{p+j+1} d x
\end{aligned}
$$

for $1 \leq j \leq p$ where $v_{0}=v_{2 p+1}$, and 


$$
\begin{aligned}
\int_{\Omega} v_{j}^{2} d x & =\int_{\Omega} v_{j-1} v_{j+1} d x=\ldots=\int_{\Omega} v_{2 j-2 p-1} v_{2 p+1} d x \\
& =\int_{\Omega} v_{2 j-2 p-2} v_{1} d x=\ldots=\int_{\Omega} v_{j-p} v_{j-p-1} d x
\end{aligned}
$$

for $p+2 \leq j \leq 2 p+1$ where $v_{2 p+2}=v_{1}$. Thus we immediately get (2.9).

Re mark 2.1. When $m=2$ Theorem 2.1 was proved by R. C. A. M. van der Vorst [9].

As an application we consider the eigenvalue problem for the polyharmonic operator:

$$
\begin{cases}(-1)^{m} \Delta^{m} u=\lambda u & \text { in } \Omega \\ u=\Delta u=\ldots=\Delta^{m-1} u=0 & \text { on } \partial \Omega .\end{cases}
$$

Then we have the following corollary.

Corollary 2.1. Problem (2.10) has a solution $u>0$ in $\Omega$ if and only if $\lambda=\mu_{1}^{m}$. The solution is given by $u=C \varphi_{1}$ where $C>0$.

Pr o of. Clearly $\lambda>0$ if $u$ is a nontrivial solution of (2.10). Now we note that problem (2.10) is equivalent to

$$
\begin{cases}-\Delta u_{j}=u_{j+1}, \quad j=1, \ldots, m-1, & \text { in } \Omega, \\ -\Delta u_{m}=\lambda u_{1} & \text { in } \Omega, \\ u_{j}=0, \quad j=1, \ldots, m, & \text { on } \partial \Omega,\end{cases}
$$

where $u=u_{1}$. Then the Maximum Principle implies that $u_{m}>0$ in $\Omega$ and by an induction argument we get $u_{j}>0$ in $\Omega$ for $j=2, \ldots, m$. Therefore we can apply Theorem 2.1.

Remark 2.2. Let $(\lambda, u)$ be a solution of (2.10) with $u \not \equiv 0$. As before we write (2.10) as (2.11) with $u=u_{1}$. Then, applying Lemma 3.9 of [9] we get

$$
m \lambda \int_{\Omega} u_{1}^{2} d x=\frac{1}{2} \int_{\partial \Omega}\left(\sum_{1 \leq j \leq m} \nabla u_{j} \cdot \nabla u_{m-j+1}\right)(\nu \cdot x) d s
$$

where $\nu$ is the outward normal on $\partial \Omega$. Then after normalizing $u$ so that $\|u\|=1$ in $L^{2}(\Omega)$ we obtain

$$
\lambda=\frac{(-1)^{m-1}}{2 m} \int_{\partial \Omega}\left(\sum_{1 \leq j \leq m} \nabla \Delta^{j-1} u \cdot \nabla \Delta^{m-j} u\right)(\nu \cdot x) d s .
$$

When $m=1$, this relation was found by F. Rellich [7].

Now if $u>0$ in $\Omega$, by Theorem 2.1 we have $u_{j}=c_{j} \varphi_{1}$ where $c_{1}>0$ is an arbitrary constant and $c_{j}=c_{1} \lambda^{(j-1) / m}$ for $j=2, \ldots, m$. We deduce 
from (2.12) that

$$
\mu_{1}=\lambda^{1 / m}=\frac{1}{2} \frac{\int_{\partial \Omega}|\nabla u|^{2}(\nu \cdot x) d s}{\int_{\Omega} u^{2} d x} .
$$

3. Proof of Theorem 1.1. We shall prove that there exists $M>0$ such that

$$
\left\|u_{j}\right\|_{\infty} \leq M, \quad j=1, \ldots, m,
$$

for all positive solutions $\left(u_{1}, \ldots, u_{m}\right) \in\left(C^{2}([a, b])\right)^{m}$ of $(1.3)$.

Let $\varphi_{1} \in C^{2}([a, b])$ be a positive eigenfunction associated with $\mu_{1}$. By $\left(\mathrm{H}_{2}\right)$, there exist $K_{j}>0$ for $j=1, \ldots, m$ such that

$$
f_{j}(u) \geq \alpha_{j} u-K_{j} \quad \text { for } u \geq 0 \text { and } j=1, \ldots, m .
$$

Now let $\left(u_{1}, \ldots, u_{m}\right) \in\left(C^{2}([a, b])\right)^{m}$ be a positive solution of (1.3). Then, $C$ denoting a generic positive constant, we have

$$
\begin{aligned}
\mu_{1}^{m} \int_{a}^{b} t^{n-1} \varphi_{1} u_{1} d t=-\mu_{1}^{m-1} \int_{a}^{b} t^{n-1} u_{1} \Delta \varphi_{1} d t \\
\quad=-\mu_{1}^{m-1} \int_{a}^{b} t^{n-1} \varphi_{1} \Delta u_{1} d t=\mu_{1}^{m-1} \int_{a}^{b} t^{n-1} \varphi_{1} f_{2}\left(u_{2}\right) d t \\
\geq \alpha_{2} \mu_{1}^{m-1} \int_{a}^{b} t^{n-1} \varphi_{1} u_{2} d t-C \geq \ldots \\
\geq \alpha_{2} \ldots \alpha_{j} \mu_{1}^{m-j} \int_{a}^{b} t^{n-1} \varphi_{1} f_{j+1}\left(u_{j+1}\right) d t-C \\
\geq \alpha_{2} \ldots \alpha_{j+1} \mu_{1}^{m-j} \int_{a}^{b} t^{n-1} \varphi_{1} u_{j+1} d t-C
\end{aligned}
$$

for $j=1, \ldots, m$ where $f_{m+1}=f_{1}, \alpha_{m+1}=\alpha_{1}$ and $u_{m+1}=u_{1}$. From (3.2) we deduce that

$$
\int_{a}^{b} \varphi_{1} u_{j} d t \leq C \quad \text { and } \quad \int_{a}^{b} \varphi_{1}\left|f_{j}\left(u_{j}\right)\right| d t \leq C
$$

for $j=1, \ldots, m$ where $C$ is again a generic positive constant. Now we have

$$
u_{j}(t)=\int_{a}^{b} G(t, s) f_{j+1}\left(u_{j+1}(s)\right) d s, \quad j=1, \ldots, m,
$$

for $t \in[a, b]$, where $G(t, s)$ denotes the Green's function of the operator - $\Delta$ on $(a, b)$ with Dirichlet boundary conditions. We easily show that 


$$
\begin{aligned}
G(t, s)=\frac{s}{(n-2) t^{n-2}\left(b^{n-2}-a^{n-2}\right)} \\
\times \begin{cases}\left(s^{n-2}-a^{n-2}\right)\left(b^{n-2}-t^{n-2}\right), & a \leq s \leq t \leq b, \\
\left(t^{n-2}-a^{n-2}\right)\left(b^{n-2}-s^{n-2}\right), & a \leq t \leq s \leq b,\end{cases}
\end{aligned}
$$

if $n \geq 3$, and

$$
G(t, s)=\frac{s}{\ln b-\ln a} \begin{cases}(\ln b-\ln t)(\ln s-\ln a), & a \leq s \leq t \leq b, \\ (\ln t-\ln a)(\ln b-\ln s), & a \leq t \leq s \leq b,\end{cases}
$$

if $n=2$. Define

$$
\varrho(t)=(t-a)(b-t) \quad \text { for } a \leq t \leq b .
$$

Then we have

$$
0 \leq G(t, s) \leq c_{1} \varrho(s) \quad \text { for } a \leq t, s \leq b
$$

and

$$
c_{2} \varrho \leq \varphi_{1} \leq c_{3} \varrho \quad \text { on }[a, b]
$$

for some positive constants $c_{j}, j=1,2,3$. From (3.3)-(3.6) we easily get $u_{j}(t) \leq C$ for $t \in[a, b]$ and $j=1, \ldots, m$ where $C$ is a positive constant, and (3.1) is proved.

\section{Proof of Theorem 1.2.}

We shall prove that problem (1.3) has at least one positive solution $\left(u_{1}, \ldots, u_{m}\right) \in\left(C^{2}([a, b])\right)^{m}$. The proof makes use of a fixed point theorem originally due to Krasnosel'skiı [5] and Benjamin [1]. Here we use the following modified version.

Proposition 4.1 ([3], p. 56). Let $C$ be a cone in a Banach space $X$ and $\Phi: C \rightarrow C$ a compact map such that $\Phi(0)=0$. Assume that there exist numbers $0<r<R$ such that

(i) $u \neq \theta \Phi(u)$ for $\theta \in[0,1]$ and $u \in C$ such that $\|u\|=r$,

(ii) there exists a compact map $F: \bar{B}_{R} \times[0, \infty) \rightarrow C$ (where $B_{\varrho}=$ $\{u \in C:\|u\|<\varrho\})$ such that $F(u, 0)=\Phi(u)$ for $\|u\|=R, F(u, x) \neq u$ for $\|u\|=R$ and $0 \leq x<\infty$ and $F(u, x)=u$ has no solution $u \in \bar{B}_{R}$ for $x \geq x_{0}$.

Then if $U=\{u \in C: r<\|u\|<R\}$, one has

$$
i_{C}\left(\Phi, B_{R}\right)=0, \quad i_{C}\left(\Phi, B_{r}\right)=1, \quad i_{C}(\Phi, U)=-1,
$$

where $i_{C}(\Phi, W)$ denotes the fixed point index of $\Phi$ on $W$. In particular, $\Phi$ has a fixed point in $U$.

Now let $X$ denote the Banach space $(C([a, b]))^{m}$ endowed with the norm

$$
\|u\|=\max _{1 \leq j \leq m}\left(\left\|u_{j}\right\|_{\infty}\right)
$$


where $u=\left(u_{1}, \ldots, u_{m}\right)$. Define the cone

$$
C=\{u \in X: u \geq 0\}
$$

where $u=\left(u_{1}, \ldots, u_{m}\right) \geq 0$ means that $u_{j} \geq 0$ for $j=1, \ldots, m$. For $(u, x) \in C \times[0, \infty)$ we define

$$
F(u, x)(t)=\left(F_{2}(u, x)(t), \ldots, F_{m}(u, x)(t), F_{1}(u, x)(t)\right) \quad \text { for } t \in[a, b]
$$

where

and

$$
F_{j}(u, x)(t)=\int_{a}^{b} G(t, s) f_{j}\left(u_{j}(s)+x\right) d s
$$

$$
\Phi(u)=F(u, 0) .
$$

We shall show that the hypotheses of Proposition 4.1 are satisfied. By $\left(\mathrm{H}_{3}\right)$ and (3.5), $F$ maps $C \times[0, \infty)$ into $C$. Since $G$ is continuous, it is wellknown that $F$ is compact. $\left(\mathrm{H}_{1}\right),\left(\mathrm{H}_{3}\right)$ and $\left(\mathrm{H}_{4}\right)$ imply that $f_{j}(0)=0$ for $j=1, \ldots, m$, hence $\Phi(0)=0$.

By $\left(\mathrm{H}_{4}\right)$ we can choose $r>0$ such that $f_{j}(s) \leq \beta_{j} s$ for $0 \leq s \leq r$ and $j=1, \ldots, m$. Suppose that there exist $\theta \in[0,1]$ and $u \in C$ with $\|u\|=r$ such that $u=\theta \Phi(u)$. Then

$$
\begin{cases}-\Delta u_{j}=\theta f_{j+1}\left(u_{j+1}\right), \quad j=1, \ldots, m-1, & \text { in }(a, b), \\ -\Delta u_{m}=\theta f_{1}\left(u_{1}\right), & \text { in }(a, b), \\ u_{j}(a)=u_{j}(b)=0, \quad j=1, \ldots, m . & \end{cases}
$$

By the Maximum Principle, for each $j \in\{1, \ldots, m\}, u_{j}>0$ on $(a, b)$ or $u_{j} \equiv 0$ on $[a, b]$. Now, if there exists $i \in\{1, \ldots, m\}$ such that $u_{i} \equiv 0$ on $[a, b]$, we easily show that $u_{j} \equiv 0$ on $[a, b]$ for $j=1, \ldots, m$. Thus $u_{j}>0$ on $(a, b)$ for $j=1, \ldots, m$. With the notations of Section 3 we have

$$
\begin{aligned}
& \mu_{1}^{m} \int_{a}^{b} t^{n-1} \varphi_{1} u_{1} d t=-\mu_{1}^{m-1} \int_{a}^{b} t^{n-1} u_{1} \Delta \varphi_{1} d t \\
& =-\mu_{1}^{m-1} \int_{a}^{b} t^{n-1} \varphi_{1} \Delta u_{1} d t=\mu_{1}^{m-1} \theta \int_{a}^{b} t^{n-1} \varphi_{1} f_{2}\left(u_{2}\right) d t \\
& \leq \beta_{2} \mu_{1}^{m-1} \int_{a}^{b} t^{n-1} \varphi_{1} u_{2} d t \leq \ldots \leq \beta_{1} \ldots \beta_{m} \int_{a}^{b} t^{n-1} \varphi_{1} u_{1} d t
\end{aligned}
$$

and we reach a contradiction because the integrals are nonzero. Thus condition (i) of Proposition 4.1 is satisfied.

By $\left(\mathrm{H}_{2}\right)$, there exists $x_{0}>0$ such that

$$
\begin{aligned}
f_{j}(s+x) \geq \alpha_{j}(s+x) \geq & \alpha_{j} s \\
& \text { for } s \geq 0, x \geq x_{0}>0 \text { and } j=1, \ldots, m .
\end{aligned}
$$


We shall show that

$$
F(u, x) \neq u \quad \text { for all } u \in C \text { and } x \geq x_{0} .
$$

Indeed, suppose that there exist $u \in C$ and $x \geq x_{0}$ such that $F(u, x)=u$. Then

$$
\begin{cases}-\Delta u_{j}(t)=f_{j+1}\left(u_{j+1}(t)+x\right), \quad j=1, \ldots, m-1, & t \in(a, b), \\ -\Delta u_{m}(t)=f_{1}\left(u_{1}(t)+x\right), & t \in(a, b), \\ u_{j}(a)=u_{j}(b)=0, \quad j=1, \ldots, m & \end{cases}
$$

If $u \equiv 0$ then $f_{j}(x)=0$ for $j=1, \ldots, m$, a contradiction to (4.1). Thus $u \not \equiv 0$. Therefore $u_{j}>0$ in $(a, b)$ for $j=1, \ldots, m$ as before. Now with the notations of the proof of (3.1) we have

$$
\begin{gathered}
\mu_{1}^{m} \int_{a}^{b} t^{n-1} \varphi_{1} u_{1} d t=-\mu_{1}^{m-1} \int_{a}^{b} t^{n-1} u_{1} \Delta \varphi_{1} d t \\
=-\mu_{1}^{m-1} \int_{a}^{b} t^{n-1} \varphi_{1} \Delta u_{1} d t=\mu_{1}^{m-1} \int_{a}^{b} t^{n-1} \varphi_{1} f_{2}\left(u_{2}+x\right) d t \\
\geq \alpha_{2} \mu_{1}^{m-1} \int_{a}^{b} t^{n-1} \varphi_{1} u_{2} d t \geq \ldots \geq \alpha_{1} \ldots \alpha_{m} \int_{a}^{b} t^{n-1} \varphi_{1} u_{1} d t
\end{gathered}
$$

and this yields a contradiction because the integrals are nonzero. Thus (4.2) holds and the third condition of (ii) is satisfied.

Now we note that the constant in (3.1) can be chosen independently of the parameter $x \in\left[0, x_{0}\right]$ for each fixed $x_{0} \in(0, \infty)$ if we consider positive solutions of (1.3) for the family of nonlinearities $f_{j, x}(t)=f_{j}(t+x), t \geq 0$. Thus we can find a constant $R>r$ such that

$$
F(u, x) \neq u \quad \text { for all } x \in\left[0, x_{0}\right] \text { and } u \in C \text { with }\|u\|=R .
$$

Therefore (4.2) and (4.3) prove the second condition of (ii).

Thus we may apply Proposition 4.1 to conclude that $\Phi$ has a nontrivial fixed point $u \in C$. Using the same arguments as before we can show that any nontrivial fixed point of $\Phi$ in $C$ yields a positive solution of (1.3) in $\left(C^{2}([a, b])\right)^{m}$. The proof of the theorem is complete.

5. Proof of Theorem 1.3. Since the proof makes use of similar arguments we only prove (i). Let $\left(u_{1}, \ldots, u_{m}\right) \in\left(C^{2}(\overline{\Omega(a, b)})\right)^{m}$ be a positive solution of (1.1), whether radial or not. With the notations of Section 3 we show as before that

$$
\mu_{1}^{m} \int_{\Omega} \varphi_{1} u_{1} d x=-\mu_{1}^{m-1} \int_{\Omega} u_{1} \Delta \varphi_{1} d x
$$




$$
\begin{aligned}
& =-\mu_{1}^{m-1} \int_{\Omega} \varphi_{1} \Delta u_{1} d x=\mu_{1}^{m-1} \int_{\Omega} \varphi_{1} f_{2}\left(u_{2}\right) d x \\
& >\gamma_{2} \mu_{1}^{m-1} \int_{\Omega} \varphi_{1} u_{2} d x>\ldots>\gamma_{1} \ldots \gamma_{m} \int_{\Omega} \varphi_{1} u_{1} d x
\end{aligned}
$$

and this yields a contradiction because the integrals are nonzero.

6. A qualitative result. In this section we give a qualitative result for positive solutions (whether radial or not) $\left(u_{1}, \ldots, u_{m}\right) \in\left(C^{2}(\overline{\Omega(a, b)})\right)^{m}$ of the problem

$$
\begin{cases}-\Delta u_{j}=f_{j+1}\left(u_{j+1}\right), \quad j=1, \ldots, m-1, & \text { in } \Omega(a, b), \\ -\Delta u_{m}=f_{1}\left(u_{1}\right) & \text { in } \Omega(a, b), \\ u_{j}=0 \text { on }|x|=b & \text { for } j=1, \ldots, m,\end{cases}
$$

where the functions $f_{j}$ satisfy

$\left(\mathrm{H}_{5}\right) \quad f_{j} \in C^{1}$ and $f_{j}^{\prime} \geq 0$ for $j=1, \ldots, m$.

The following theorem is an extension to systems of a theorem obtained by B. Gidas, W.-M. Ni and L. Nirenberg (see [4], Theorem 2, p. 210).

THEOREM 6.1. Assume $\left(\mathrm{H}_{5}\right)$. Let $\left(u_{1}, \ldots, u_{m}\right) \in\left(C^{2}(\overline{\Omega(a, b)})\right)^{m}$ be a positive solution of (6.1). Then

$$
x \cdot \nabla u_{j}(x)<0 \quad \text { for } \frac{a+b}{2} \leq|x|<b \text { and } j=1, \ldots, m .
$$

Proof. The proof makes use of a lemma proved by W. C. Troy ([8], Lemma 4.3, p. 408). We note that, with the notations of Lemma 4.3 in [8], the condition $u_{i} \equiv 0$ on $\partial \Omega$ for $i=1, \ldots, n$ is not needed: we only need $u_{i} \equiv 0$ on $\partial \Omega \cap\left\{x \in \mathbb{R}^{n}: x_{1}>\lambda_{1}\right\}$ for $i=1, \ldots, n$. Then the proof is the same as in the scalar case (see [4]).

Remark 6.1. Note that, as in the scalar case, no condition is imposed on the boundary $|x|=a$ and the result holds without any sign condition on the $f_{j}$.

\section{References}

[1] T. B. Benjamin, A unified theory of conjugate flows, Philos. Trans. Roy. Soc. 269 A (1971), 587-643.

[2] Ph. Clément, D. G. de Figueiredo and E. Mitidieri, Positive solutions of semilinear elliptic systems, Comm. Partial Differential Equations 17 (1992), 923-940.

[3] D. G. de Figueiredo, P.-L. Lions and R. D. Nussbaum, A priori estimates and existence of positive solutions of semilinear elliptic equations, J. Math. Pures Appl. 61 (1982), 41-63. 
[4] B. Gidas, W.-M. Ni and L. Nirenberg, Symmetry and related properties via the maximum principle, Comm. Math. Phys. 68 (1979), 209-243.

[5] M. A. Krasnosel'ski ̌, Fixed points of cone-compressing and cone-extending operators, Soviet Math. Dokl. 1 (1960), 1285-1288.

[6] L. A. Peletier and R. C. A. M. van der Vorst, Existence and non-existence of positive solutions of non-linear elliptic systems and the biharmonic equation, Differential Integral Equations 5 (1992), 747-767.

[7] F. Rellich, Darstellung der Eigenwerte von $\Delta u+\lambda u=0$ durch ein Randintegral, Math. Z. 46 (1940), 635-636.

[8] W. C. Troy, Symmetry properties in systems of semilinear elliptic equations, J. Differential Equations 42 (1981), 400-413.

[9] R. C. A. M. van der Vorst, Variational identities and applications to differential systems, Arch. Rational Mech. Anal. 116 (1991), 375-398.

LABORATOIRE LMC-IMAG

EQUIPE EDP

TOUR IRMA

B.P. $53 \mathrm{X}$

F-38041 GRENOBLE CEDEX, FRANCE

E-mail: DALMASSO@IMAG.FR 